\title{
Cost-Effectiveness Analysis of Raltegravir in Treatment-Experienced HIV Type 1-Infected Patients in Spain
}

\author{
Mohammad A. Chaudhary, Santiago Moreno, ${ }^{2}$ Ritesh N. Kumar, ${ }^{3}$ Gonzalo Nocea, ${ }^{4}$ and Elamin Elbasha ${ }^{1}$
}

\begin{abstract}
Raltegravir, a novel HIV-1 integrase inhibitor, has superior efficacy with optimized background treatment (OBT) vs. placebo + OBT in treatment-experienced HIV-1 patients. This study assessed the long-term cost effectiveness of raltegravir from a Spanish National Healthcare System perspective. A cohort-state-transition model was used to estimate clinical and economic outcomes associated with raltegravir + OBT vs. OBT alone. Subjects were stratified into health states according to HIV RNA level, CD4 count, and opportunistic infection (OI) history, and could transition into different health states over time based on projected long-term efficacy. Each health state was associated with a distinct treatment cost and utility (QoL) score. Model inputs for mortality, resource utilization, unit costs, OI risk, and long-term durability of viral suppression were obtained from clinical trials, published studies, and database analyses. Model outcomes were reported as incremental cost-effectiveness ratios (ICERs) in 2007 Euros per quality-adjusted life-year (€/QALY) gained. Costs and QALYs were discounted at 6\% per year based on Spanish cost-effectiveness guidelines. Extensive sensitivity analyses were conducted. Five years of treatment with raltegravir + OBT resulted in an additional 4.5 years of undiscounted life expectancy vs. OBT alone. The ICER of raltegravir + OBT vs. OBT alone was €22,908/QALY and €31,431/QALY for 3- and 5 -year use, respectively. Lower ICERs were observed with lower discount rates (3\%) for costs and benefits, lower raltegravir price $(20 \%)$, and shorter treatment duration (3 years). ICER was also sensitive to analytical time horizon and alternative sources of QoL scores. In treatment-experienced Spanish patients, raltegravir was projected to provide survival benefits and be cost effective.
\end{abstract}

\section{Introduction}

D ESPITE THE SUCCESS OF CURRENT and emerging antiretroviral therapies (ARTs) in reducing HIV viral load and decreasing morbidity and mortality for HIV-infected individuals, ${ }^{1,2}$ the ability to sustain long-term suppression of viral turnover is increasingly compromised by the development of resistance to existing agents. ${ }^{3}$ Over time, patients with advanced HIV infection are likely to experience treatment exhaustion; one estimate suggests that $20 \%$ of such patients develop triple-class failure within 6 years. ${ }^{4}$ In patients with resistant disease, the use of novel ART agents can offer considerable potential benefit because of the absence of crossresistance. $^{5}$
Raltegravir (Isentress, Merck \& Co., Inc.) is an inhibitor of human immunodeficiency virus type 1 (HIV-1) integrase, effective against strains of HIV-1 including those resistant to older antiretroviral drugs. ${ }^{5,6}$ Two parallel multicenter, randomized clinical trials (BENCHMRK-1 and BENCHMRK-2) have demonstrated the potent efficacy and good tolerability of raltegravir $400 \mathrm{mg}$ bid vs. placebo when used as an adjunct to optimized background therapy (OBT). ${ }^{6,7}$ For example, suppression of HIV-1 RNA to a level $<50$ copies $/ \mathrm{ml}$ was achieved in $61.8 \%$ of raltegravir recipients compared with $34.7 \%$ of placebo recipients at week 16 and in $62.1 \%$ of raltegravir recipients compared with $32.9 \%$ of placebo recipients at week 48 ( $p<0.001$ for both comparisons). Raltegravir was generally well tolerated through 48 weeks, with few drug-related

\footnotetext{
${ }^{1}$ Merck Research Laboratories, North Wales, Pennsylvania 19454.

${ }^{2}$ Hospital Ramón y Cajal, Madrid, Spain.

${ }^{3}$ Merck \& Co., Inc., Whitehouse Station, New Jersey 08889.

${ }^{4}$ Merck Sharp \& Dohme, Madrid, Spain.
} 
discontinuations. ${ }^{6}$ Virologic responses to raltegravir were consistently superior to responses to placebo, regardless of the baseline HIV-1 RNA level; CD4 cell count; genotypic or phenotypic sensitivity score; use or nonuse of darunavir, enfuvirtide, or both in optimized background therapy; or demographic characteristics. ${ }^{7}$ Raltegravir is currently licensed in Europe for use in combination with other ARTs in treatmentexperienced HIV-1-infected patients with evidence of ongoing viral replication despite ART. ${ }^{8}$

The cost of treating HIV-related diseases has been increasing, but HIV/AIDS infection also results in high indirect costs over time for individual patients: in Spain, the annual loss of income is estimated at $€ 5,271$ to $€ 6,150$ per patient and loss of wages ranges from $€ 7,537$ to $€ 8,793$. ${ }^{9}$ Pharmacologic treatment has the potential to reduce these costs and result in higher quality of life (QoL). ${ }^{9}$

The rising cost of medical care also means that reimbursement decisions about new therapies are increasingly based on their relative cost effectiveness as determined by pharmacoeconomic evaluations. Cost effectiveness for traditional HIV treatments has been well researched ${ }^{10,11}$ and has become instrumental in demonstrating value to payors. This study examines the likely effect of adding raltegravir to OBT on medical costs, life expectancy, and quality-adjusted life expectancy from a Spanish National Healthcare System perspective. The results were used to calculate the incremental cost-effectiveness ratio (ICER) as the incremental cost per quality-adjusted life-year (QALY) gained with raltegravir + OBT vs. OBT alone.

\section{Materials and Methods}

A cohort state-transition model was used to evaluate the long-term health outcomes for treatment-experienced patients receiving raltegravir $400 \mathrm{mg}$ bid + OBT or OBT alone. A detailed description of the model and inputs is included in the accompanying technical appendix. Patients were stratified by health states, as defined by HIV RNA level, CD4 count, history of opportunistic infection (OI), and temporary OI status with each state associated with a specific treatment cost and QoL score. Clinical and economic inputs were determined using data from published clinical trials and database analyses. ICERs were expressed as Euros per quality-adjusted life-year (€/QALY) gained. Cost-effectiveness analyses were conducted in accordance with the recommendations of the Panel on Cost-Effectiveness in Health and Medicine for conducting and reporting a reference-case analysis and Spanish pharmacoeconomic guidelines. ${ }^{12-15}$

\section{Target population}

A hypothetical cohort of patients was generated representing the target population of treatment-experienced HIV-1-infected individuals resistant to at least one drug in each of the three classes [nucleoside reverse transcriptase inhibitors (NRTIs), nonnucleoside reverse transcriptase inhibitors (NNRTIs), and protease inhibitors (PIs)] of ARTs. The distribution of HIV RNA levels and CD4 cell counts among patients in the placebo and raltegravir groups was based on the baseline data of patients in the BENCHMRK trials. ${ }^{6}$

Eighty-eight percent of included patients were men, and over $90 \%$ had a diagnosis of AIDS. Other baseline characteristics included mean age (45 years), median number of ARTs used prior to the study (12), mean HIV RNA $\log _{10}$ copies $/ \mathrm{ml}$ (4.7), and median CD4 cell count $(102 / \mu 1)$. Table 1 summarizes these data by study arm.

\section{HIV disease progression economic model}

Our model, which followed previously reported costeffectiveness analyses for HIV treatments, used a cohort statetransition modeling approach that assumed a Markov process with limited history. ${ }^{16-21}$ However, the raltegravir model differed in that it was a continuous-time state transition model developed in Mathematica 6.0 (Wolfram Research, Inc., Champaign, IL). It was thus able to account for health states at every moment in time and allowed for events to occur at any moment in time, rendering the length of the cycle infinitesimally small or irrelevant. Movements between health states were represented by three sets of differential equations corresponding to OI status: current OI, with history of OI, and without history of OI. The number of health states within each of these broad heath states was determined through combinations of OI type, HIV RNA level, and CD4 level. OI types considered in the model included Pneumocystis jiroveci pneumonia (PCP), disseminated Mycobacterium avium complex (MAC), toxoplasmosis, cytomegalovirus infection (CMV), Mycobacterium tuberculosis (TB), fungal infection (systemic or esophageal candidiasis, coccidiomycosis, cryptococcosis, or histoplasmosis), and "other" AIDS-related complications, such as lymphoma. Monthly death rates and the mean duration of OI episodes for patients with OIs and with a history of OIs were estimated from the literature (see Model inputs and data sources). Because of the heterogeneity and rarity of OIs, detailed data on costs and QoL were lacking and could not be accounted for according to OI types. Instead, costs and QoL were captured by the CD4 cell count group. All patients were assumed to have received standard first-line primary prophylaxis against PCP based on 2004 GESIDA/Plan Nacional sobre el SIDA guidelines for the prevention of OIs in persons infected with HIV. ${ }^{22}$ Prophylaxis for toxoplasmosis was included with prophylaxis for PCP in these guidelines.

To track viral load and CD4 cell count over time, HIVrelated health states were defined by seven HIV viral load and six CD4 cell count strata: HIV RNA level $\geq 100,000,30,001-$

TABle 1. TARget BENCHMRK Population CHARACTERISTICS

\begin{tabular}{lcc}
\hline Characteristics & Isentress + OBT & Placebo + OBT \\
\hline Number of patients & 462 & 237 \\
Mean age (SD) & $45.7(8.6)$ & $45.1(8.1)$ \\
Male, \% & 87.7 & 88.6 \\
White, \% & 65.2 & 73.0 \\
Mean CD4 count & $151.4(141.2)$ & $158.0(150.4)$ \\
$\quad$ cells/mm m $^{3}$ (SD) & & \\
Geometric mean HIV & 44,252 & 39,034 \\
$\quad$ RNA copies/ml & & \\
Mean HIV RNA log & $4.6(0.8)$ & $4.6(0.8)$ \\
$\quad$ copies/ml (SD) & & \\
History of AIDS, \% & 92.5 & 10.1 \\
Median years of prior & 10.1 & 12 \\
$\quad$ ART use & & \\
Median number of & 12 & \\
$\quad$ prior ARTs used & & \\
\hline
\end{tabular}


$100,000,10,001-30,000,3001-10,000,401-3000,51-400$, and $0-50$ copies $/ \mathrm{ml}$; and CD4 count $>500,301-500,201-300,101-$ 200, 51-100, and 0-50 cells $/ \mu \mathrm{l}$.

After the initial treatment assignment, patients were able to progress through various disease pathways, moving from a temporary health state to a new chronic health state or dying (Fig. 1). HIV-related mortality and morbidity rates were based on $\mathrm{CD} 4$ cell count.

\section{Perspective and time horizon of the analysis}

The economic analysis was conducted from a Spanish healthcare system perspective and was based on direct treatment costs. Our model assumed an analytic time horizon of 50 years for the trial cohort based on a mean entry age of 45 years. It was expected that major differences in costs and anticipated treatment-related benefits would be accounted for within the model's time horizon.

\section{Outcomes}

For each simulation, the lives of a cohort were simulated and followed until death or the fixed time horizon of 50 years was reached. The numbers of primary and recurrent OI cases and life expectancy were calculated, but because patients with higher viral loads and lower CD4 cell counts can be expected to incur greater medical costs due to a higher incidence of comorbid conditions and OIs, medical costs were allowed to vary according to $\mathrm{CD} 4$ cell count and viral load category.

QoL scores were also determined by CD4 cell count. Cumulative QALYs, cumulative expected costs, incremental costs, and QALYs associated with raltegravir + OBT vs. placebo + OBT were calculated. All costs were expressed in 2007 Euros using the Spanish consumer price index. ${ }^{23}$ Future costs and QALYs were discounted to the present value at a rate of $6 \%$ per year, as suggested by the Spanish pharmacoeconomic guidelines ${ }^{15}$; lower and higher rates were applied in the sensitivity analysis.

\section{Model inputs and data sources}

Efficacy and duration of treatment. Baseline CD4 cell counts and HIV RNA levels for patients in each arm of the BENCHMRK trials were used as initial values for the model, and rates of movement between the seven viral load categories were estimated using "transition matrices" (Supplementary Appendix available on request). The antiretroviral activity of raltegravir $+\mathrm{OBT}$ vs. placebo $+\mathrm{OBT}$ was input in two stages using baseline, week 4, and week 48 BENCHMRK trial data.

The model assumed that virologic and immunologic failure rates would remain stable for a fixed period of time at the levels observed during the first 48 weeks. It is currently unknown how long patients will be taking raltegravir. The primary analysis applied a 5-year treatment duration assumption. In the base case, we assumed that virologic and immunologic failure rates would remain stable for a period of 5 years at the levels observed during the first 48 weeks in the BENCHMRK. After year 5, the annual rate of rebound from one HIV RNA stratum to the next higher stratum was assumed to be $8 \%,{ }^{24}$ which is broadly consistent with ART discontinuation rates reported in the literature. While data inputs were based on the 48-week BENCHMRK study time- frame, the percentage of patients in the raltegravir arm with an undetectable viral load of $<50$ copies $/ \mathrm{ml}$ at 16 weeks $(62 \%), 24$ weeks $(63 \%)$, and 48 weeks $(62 \%)$ was very similar, suggesting a degree of stability over time. Nonetheless, the sensitivity analysis varied the rate of rebound (between $1 \%$ and $16 \%$ per year). When patients stopped receiving raltegravir, their rates of movement between viral load and CD4 cell categories were set to the same rates as the placebo group.

Substantial differences in the probability of discontinuation of individual drugs were observed in an analysis of the incidence of treatment change within 1 year after starting combination ART among 1866 patients from the Swiss HIV Cohort Study who initiated ART during 2000-2005. ${ }^{25}$ For example, the 12-month probability of discontinuation for NRTIs ranged from $20 \%$ for tenofovir to $51 \%$ for stavudine, while the 12-month probability of discontinuation for PIs or NNRTIs ranged from $16 \%$ for atazanavir to $43 \%$ for nevirapine. This implies an average duration of treatment of 2-10 years; shorter and longer treatment durations were assessed in the sensitivity analysis.

Movement between CD4 cell count categories. The annual rate of $\mathrm{CD} 4$ change was estimated using a regression model developed by the PLATO Collaboration, based on an analysis of data from 13 multinational cohorts of patients with HIV (viral load $>1000$ copies $/ \mathrm{ml}$ for $>4$ months). ${ }^{26,27}$ The model provides a relationship between $\mathrm{CD} 4$ cell count slope, RNA concentration, treatment, and demographic factors.

Risk of opportunistic infections. The monthly incidence of OIs and other AIDS-related complications was based on data from the Multicenter AIDS Cohort Study (MACS), an ongoing U.S.-based investigation of the natural history and treated progression of HIV-1 infection in bisexual and homosexual men. ${ }^{10,28}$ Because these data did not take the impact of ART into account, the rates were adjusted downward using relative hazard rates comparing the rates of diseases during the periods 1994-1996 and 1997-2001. ${ }^{29}$ Relative hazard rates for $\mathrm{PCP}$, TOXO, MAC, fungal infections, CMV, TB, recurrent bacterial pneumonia, and other OIs (lymphomas) were 0.22 , $0.3,0.23,0.3,0.1,0.41,0.38$, and 0.52 ; respectively. Incidence rates from other studies were used for validation. ${ }^{30-33}$

Mortality rates and duration of opportunistic infections. Since OIs can have a significant impact on a person's mortality, three broad categories of mortality rates were used in the model: all-cause mortality, mortality following an acute OI, and HIV-related mortality for patients with or without a history of OIs. The baseline mortality rates of patients with a history of OIs were derived from EuroSIDA data from the late HAART period (1998) ${ }^{34,35}$ and were adjusted according to CD4 cell count. The EuroSIDA study is a prospective European-based study made up of HIV-1 patients from 72 centers in Europe. Because approximately 25\% of patients had AIDS before enrollment for this period, the estimates from the EuroSIDA were adjusted to reflect a relative hazard of death of patients with history of OIs of 1.5. ${ }^{26}$

Monthly death rates in the presence of OIs were obtained from Moore and Chaisson ${ }^{36}$ and Chaisson et al. ${ }^{37}$ and monthly mortality rates for patients with an OI history were estimated from Moore and Chaisson, ${ }^{36}$ Chaisson et al. ${ }^{37}$ and Wohl et al., ${ }^{38}$ and validated through comparison with rates from 


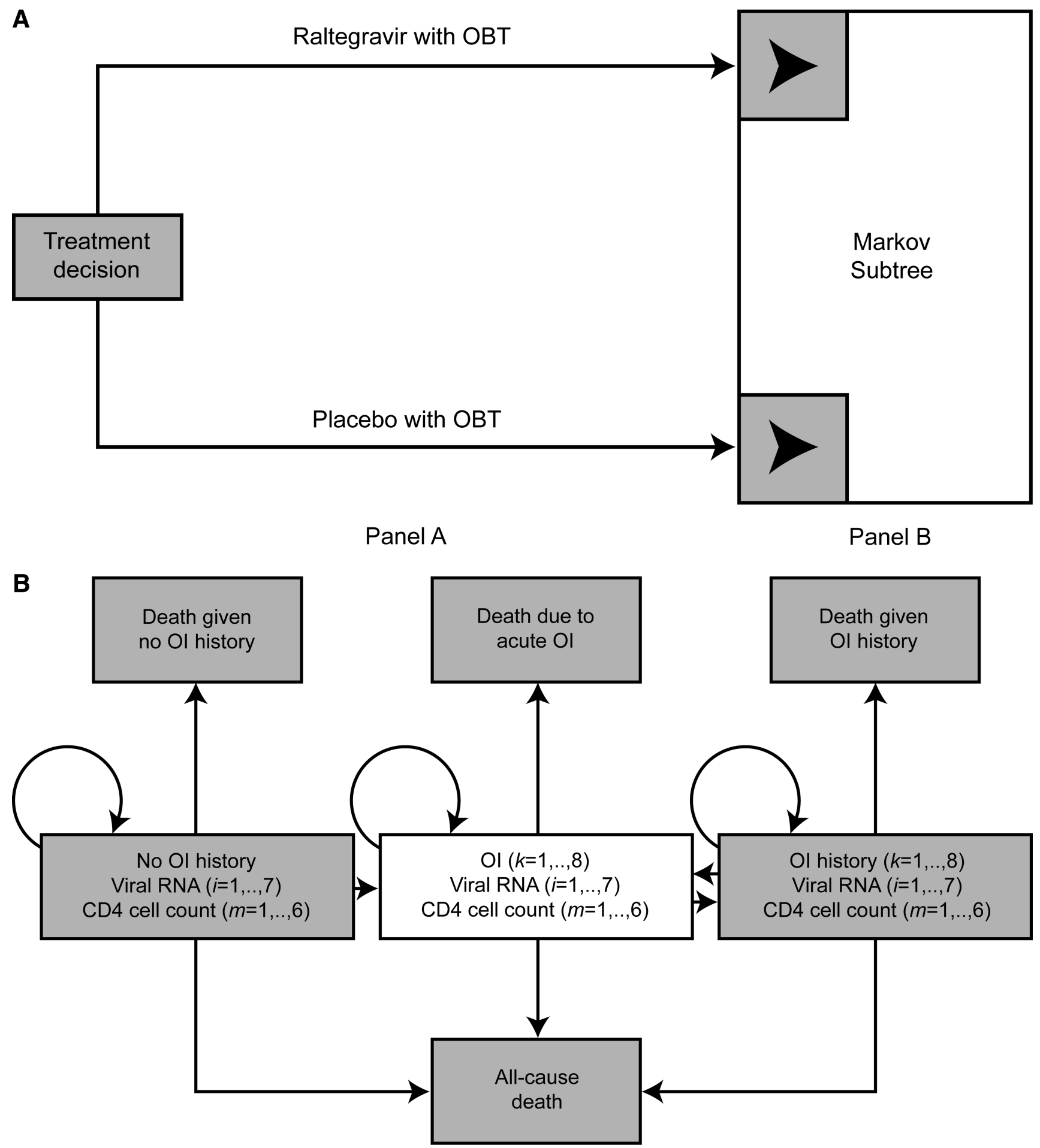

FIG. 1. (A) Schematic representation of the HIV decision analytic model: treatment strategies. The square node at the left represents the decision to treat HIV-1 patients with either raltegravir + OBT or placebo + OBT. The patient's health thereafter is simulated using a Markov model (B). OBT, optimized background therapy. (B) HIV diseases progression model. Patients may enter the model with a given HIV-1 viral load and CD4 cell count. Each month all HIV patients are at risk for moving to higher or lower viral load and lower or higher CD4 cell count states. In addition, each month patients are at risk of developing OIs. Patients who develop an opportunistic disease can either die or recover and move to the health state OI history. Throughout their lifetime, all patients are at risk for non-HIV-related mortality. OI, opportunistic infection. 
other studies. ${ }^{39}$ The duration of OI was estimated using a variety of published sources. These data are summarized in Table 2.

It is estimated that approximately $50 \%$ of deaths among HIV-1-infected patients were not related to AIDS. ${ }^{40}$ Death rates, according to age, from causes other than HIV were estimated from 2004-2005 Spanish life tables for males aged 30 years and over. ${ }^{41}$ The life table mortality data were fitted to a Gompertz distribution and age-specific mortality rates were estimated.

Costs. The analysis included direct medical costs of ART, OI prophylaxis, and other healthcare resource utilization (testing, monitoring, hospitalization, emergency, outpatient, general practitioner and specialist visits). Antiretroviral drug costs for OBT were based on the distribution of drugs in the regimen as observed within the BENCHMRK studies, and on the prices of drugs based on the daily recommended dose in Spain. If a drug was not available and marketed in Spain, its price was estimated based on the prices of other antiretroviral drugs. OBT costs should therefore be considered as rough estimates.

The weighted average cost per day for OBT was estimated at $€ 54.90$, and the daily cost of raltegravir (€27.00) was added

Table 2. Mortality Rates and Duration of OIs

\begin{tabular}{lcc}
\hline & HIV-related mortality per month & \\
\hline & $\begin{array}{c}\text { Without OI } \\
\text { history }^{\mathrm{b}}\end{array}$ & $\begin{array}{c}\text { With OI } \\
\text { history }^{\mathrm{c}}\end{array}$ \\
\hline CD4 0-50 & 0.00297 & 0.00450 \\
CD4 51-100 & 0.00083 & 0.00125 \\
CD4 101-200 & 0.00064 & 0.00096 \\
CD4 201-300 & 0.00020 & 0.00031 \\
CD4 301-500 & 0.00018 & 0.00027 \\
CD4 500 & 0.00019 & 0.00028 \\
\hline
\end{tabular}

Duration (months) of acute OI and monthly risk of death

\begin{tabular}{lcc}
\hline Opportunistic infection & Duration $^{\mathrm{d}}$ & $\begin{array}{c}\text { Risk of } \\
\text { death }^{\mathrm{e}}\end{array}$ \\
\hline $\begin{array}{l}\text { Pneumocystis jirovecii } \\
\quad \text { pneumonia (PCP) }\end{array}$ & 1.20 & 0.0511 \\
$\begin{array}{l}\text { Cerebral toxoplasmosis (TOXO) } \\
\text { Mycobacterium avium complex }\end{array}$ & 4.00 & 0.1187 \\
$\quad$ infection (MAC) & 11.30 & 0.1029 \\
$\begin{array}{l}\text { Fungal infections } \\
\text { Cytomegalovirus infection (CMV) }\end{array}$ & 0.88 & 0.0511 \\
$\begin{array}{l}\text { Pulmonary or extrapulmonary } \\
\quad \text { tuberculosis (TB) }\end{array}$ & 6.90 & 0.0804 \\
\end{tabular}

${ }^{\text {a } O t h e r ~ a l l-c a u s e ~ m o r t a l i t y ~ r a t e s ~ a r e ~ e s t i m a t e d ~ f r o m ~ t h e ~} 2005$ Spanish life tables for males aged at least 30 years. ${ }^{43}$ These mortality data were fitted to a Gompertz distribution and the resulting annual age-specific mortality rate was $\mu($ age $)=\operatorname{Exp}(-10.025+0.092 \cdot$ age $)$, where age denotes age in years.

${ }^{\mathrm{b}} \mathrm{HIV}$-related mortality in patients without OI history per month is $1.5 \%$ lower than HIV-related mortality in patients with OI history per month.

${ }^{c}$ HIV-related mortality with history of OI was obtained from Moore and Chaisson, ${ }^{36}$ Chaisson et al., ${ }^{37}$ and Wohl et al. ${ }^{38}$

${ }^{\mathrm{d}}$ Different sources were used for duration of OI: $\mathrm{TB}_{r}{ }^{64} \mathrm{PCP}^{65}$ TOXO, ${ }^{66,67} \mathrm{CMV}^{68} \mathrm{MAC}^{69}$ and fungal infections. ${ }^{70,71}$

${ }^{\mathrm{e}}$ For mortality following an OI, Moore and Chaisson ${ }^{36}$ were used except for TB. ${ }^{64}$ to the other costs in the raltegravir arm. The cost of raltegravir was based on a dose of $400 \mathrm{mg}$ bid. Although the drugs that comprised OBT were assumed to be similar for patients on placebo and raltegravir, the cost of OBT still had to be estimated for the incremental cost-effectiveness analysis, as patients on raltegravir are expected to live longer on average and thus incur more drug costs.

The base case in our model assumes 5 years of raltegravir therapy, and the drug's price and treatment durations are varied in the sensitivity analysis. Costs were not applied to specific OIs, as patients have been incurring an increasingly broad range of comorbid conditions (cancer and cardiovascular, liver and renal disease). Modeling the costs for all of these would have been prohibitively complex; the model therefore incorporated study data on resource utilization according to CD4 counts and viral load, without attributing the costs to a particular OI or comorbidity. The resource use distribution by CD4 and viral load categories was not available for Spain and was derived from the HIV database from the British Columbia Center for Excellence in HIV. ${ }^{42}$ The analysis tracked 2718 patients who initiated therapy between 1995 and 2000, and their resource utilization through March 31, 2001 in categories such as hospitalization, specialist visits, general practitioner visits, and tests. Monthly costs for HIVrelated services and laboratory work were obtained from Spain. The estimated monthly costs of resource utilization broken down by CD4 and HIV RNA levels are shown in Table 3.

Table 3. Monthly Cost of Resource Use AND HIV-RELATED LABORATORY WORK ${ }^{\mathrm{a}}$

\begin{tabular}{|c|c|}
\hline CD4/VL category & $2007 €$ \\
\hline CD4 $0-50, \mathrm{VL} \geq 100,000$ & 963 \\
\hline CD4 0-50, VL 10,001-100,000 & 761 \\
\hline CD4 0-50, VL 401-10,000 & 949 \\
\hline CD4 $0-50, \mathrm{VL} \leq 400$ & 444 \\
\hline $\mathrm{CD} 451-100, \mathrm{VL} \geq 100,000$ & 414 \\
\hline CD4 51-100, VL $10,001-100,000$ & 333 \\
\hline CD4 51-100, VL 401-10,000 & 381 \\
\hline CD4 51-100, VL $\leq 400$ & 221 \\
\hline CD4 101-200, VL $\geq 100,000$ & 411 \\
\hline CD4 101-200, VL 10,001-100,000 & 317 \\
\hline CD4 101-200, VL 401-10,000 & 261 \\
\hline CD4 101-200, VL $\leq 400$ & 167 \\
\hline CD4 201-300, VL $\geq 100,000$ & 278 \\
\hline CD4 201-300, VL $10,001-100,000$ & 230 \\
\hline CD4 201-300, VL 401-10,000 & 195 \\
\hline CD4 201-300, VL $\leq 400$ & 149 \\
\hline $\mathrm{CD} 4>300, \mathrm{VL} \geq 1 \overline{0} 0,000$ & 247 \\
\hline CD4 > 300, VL $\overline{1} 0,001-100,000$ & 192 \\
\hline CD4 > 300, VL 401-10,000 & 164 \\
\hline $\mathrm{CD} 4>300, \mathrm{VL} \leq 400$ & 126 \\
\hline
\end{tabular}

a Based on the resource use distribution by $\mathrm{CD} 4$ and viral load categories derived from the HIV database from the British Columbia Center for Excellence in HIV ${ }^{44}$ and the monthly costs of services and HIV-related laboratory work obtained from Spain. Resource use by HIV patients included treatment for opportunistic infections and ART. The following per month costs from Spain were used: inpatient day (non-ICU): €260.11; inpatient day (ICU) €1013.71; general practitioner visit $€ 23.56$; specialist visit $€ 58.29$; ER visit $€ 119.02$; CD4 cell count test €7.59; HIV-1 RNA test €52.52; and genotypic resistance test $€ 150$. 
Because the BENCHMRK trials tested raltegravir as an add-on therapy and showed no evidence of adverse events (AEs) related specifically to raltegravir, this analysis did not include costs associated with the management of AEs.

Quality of life. QoL scores distributed between 1 (best possible health state) and 0 (death) were assigned to each HIV health state based on a previously published study that analyzed the responses of 21,000 patients to questions in the EuroQoL quality of life instrument, EQ-5D. ${ }^{43,44}$ These estimates was used in the base case analysis because of the large sample size, recent data collection, and the stratification of QALY scores by CD4 cell count and VL categories. However, the scores may not be reflective of highly treatmentexperienced patients as the lowest utility value was 0.781 for a CD4 count of $0-50$ cells / $\mu$ l and any viral load category and the highest was 0.954 for a CD4 count of $>500$ cells $/ \mathrm{mm}^{3}$ and a viral load of $\leq 400$ copies $/ \mathrm{ml}$ (Table 4 ). Because of variations in estimates of utility scores, ${ }^{45}$ sensitivity analysis also included utilities derived from other sources that were lower and higher than those used in the base case analysis. ${ }^{46-48}$ The utility values reported by Stavem et al. were lower than those used in the base case, ranging from 0.55 to 0.86 for CD4 counts of $0-50$ and $>500$ cells $/ \mu$ l, respectively. ${ }^{48}$

\section{Validation and sensitivity analysis}

Face validity of the model was established through consultation with HIV experts on assumptions relating to the natural history of HIV infection, disease process, treatment, and testing patterns. The model's predictive validity was determined by comparing results to data from the trials, cohort studies, and antiretroviral drug intervention studies. ${ }^{26,49,50}$

One-way sensitivity analyses were performed using the following parameters: analytic horizon of the analysis, discount rate, QoL weights, price of raltegravir, cost of OBT, duration of raltegravir treatment, and treatment failure rates. When duration of treatment was varied, viral load transitional probabilities were also varied, such that patients in the 10-year analysis received the same transitional probabilities as OBT and no additional raltegravir costs after 10 years. Multiway sensitivity analyses were also conducted by varying the

Table 4. Health-Related Quality of Life Scores by Health States

\begin{tabular}{lc}
\hline CD4 $/$ HIV RNA categories & QoL scores $^{\mathrm{a}}$ \\
\hline CD4 0-50, all viral load levels & 0.781 \\
CD4 51-200, V $>100,000$ & 0.826 \\
CD4 51-200, V 30,001-100,000 & 0.856 \\
CD4 51-200, V 401-30,000 & 0.865 \\
CD4 51-200, V $<400$ & 0.863 \\
CD4 201-300, V $>30,000$ & 0.933 \\
CD4 201-300, V 401-30,000 & 0.931 \\
CD4 201-300, V $<400$ & 0.929 \\
CD4 301-500, V $>400$ & 0.931 \\
CD4 301-500, V $\leq 400$ & 0.934 \\
CD4 $>500, V>400$ & 0.938 \\
CD4 $>500, V \leq 400$ & 0.954
\end{tabular}

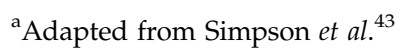

risk of developing OIs and the sources of data for mortality rates and estimates of health utility weights.

\section{Results}

\section{Model validation}

Projected model outcomes were generally within the range of values reported in the literature, and model-based prediction of overall mortality also compared well. The model prediction of the annual rate of death in the first year of simulation was $4.9 \%$, similar to that estimated by cohort studies. For example, in the PLATO Collaboration study of patients who had had three-class virological failure, 267 patients died during 5015 person-years of follow up (PYFU), resulting in a mortality rate of 5.5 per 100 PYFU. ${ }^{26}$ Populationbased estimates of mortality rates in 2000 and 2001 from the Adult and Adolescent Spectrum of HIV Disease (ASD) surveillance were 5.7 and 4.9 per 100 PYFU, respectively, ${ }^{51}$ whereas annual mortality within the Swiss HIV Cohort Study after 1998 was $3 \% .^{52}$ The model prediction of the average annual rate of death during 50 years was $1.7 \%$, which is slightly higher than in recent studies mainly because of differences in the population characteristic between these studies and the model (e.g., we assumed a lower baseline CD4 cell count, a higher HIV RNA level, and over $90 \%$ with prior AIDS diagnosis). For example, the estimated annual rate of death obtained using data from the Antiretroviral Therapy Cohort Collaboration was $1.42^{53}$ and similar rates were estimated using data from the HIV Outpatient Study (HOPS). ${ }^{39}$ The death rates among the 6945 patients in HOPS declined from 7.0 per 100 PYFU in 1996 to 1.3 per 100 PYFU in 2004. The death rate among 5122 patients with 12,246 PYFU in the CASCADE Collaboration was 1.6 per 100 PYFU during the period 1999-2001, ${ }^{54}$ whereas the rate of death from any cause among the patients in the Drug Conservation Group of the SMART trial was $1.5 \%$ per year. ${ }^{55}$ Projected outcomes of opportunistic disease generally fell within the range reported in the literature.

\section{Base case analysis}

Table 5 shows the total discounted costs, life expectancy, quality-adjusted life expectancy, and ICER associated with raltegravir $+\mathrm{OBT}$ vs. placebo $+\mathrm{OBT}$ for an analytical time horizon of 50 years and a treatment duration of 5 years. Raltegravir increased undiscounted life expectancy by 4.52 years at an incremental cost of $€ 117,637$ vs. OBT alone (not shown). After discounting both costs and QALYs at 6\%, the incremental QALYs and costs are 1.72 years and $€ 53,981$, respectively, resulting in an ICER of $€ 31,431 /$ QALY.

\section{Sensitivity analysis}

The results were robust to changes in several individual inputs values (Fig. 2), but they were most sensitive to raltegravir treatment duration, OBT costs, analytic time horizon, and raltegravir cost. The greatest impact on ICER was observed when raltegravir treatment duration was varied from 3 years (ICER $=€ 22,908 / \mathrm{QALY}$ ) to lifetime raltegravir use (ICER $=€ 74,468 / \mathrm{QALY})$, suggesting that longer use of raltegravir resulted in more drug costs and a higher ICER. The time horizon of the analysis is another influential input: the shorter the analytic horizon, the fewer the differential benefits 
Table 5. Incremental Cost Effectiveness of Raltegravir + OBT vs. Placebo + ObT: Base-Case Scenario ${ }^{a}$

\begin{tabular}{lccc}
\hline Strategy & $\begin{array}{c}\text { Undiscounted } \\
\text { life expectancy } \\
\text { (years) }\end{array}$ & $\begin{array}{c}\text { Discounted } \\
\text { QALYS } \\
\text { (years) }\end{array}$ & $\begin{array}{c}\text { Discounted } \\
\text { total cost }(€)\end{array}$ \\
\hline Placebo + OBT & 24.65 & 10.18 & 275,509 \\
Isentress + OBT & 29.17 & 11.90 & 329,490 \\
\hline
\end{tabular}

${ }^{a}$ Data reported here are based on an analytic horizon of 50 years and a 5-year duration of raltegravir therapy; OBT, optimized background therapy; QALY, quality-adjusted life-year. Costs and QALYs were discounted at $6 \%$ per year.

of raltegravir and the higher the ICER (and vice versa). Varying incidence of OIs, discount rate, QoL decrement, and future placebo and raltegravir failure rate also had a modest impact on ICER.

The multiway sensitivity analysis suggested that the results are also sensitive to variations in the QoL data from alternative sources (Table 6). We found that when QoL weights were used that varied very little by viral load and CD4 status, the ICER was higher. Using HIV-related mortality data from Mocroft et al., ${ }^{34}$ increasing OI risk or eliminating it altogether had a modest impact on ICER.

\section{Discussion}

There is no currently accepted threshold for cost effectiveness among ARTs. A frequently-cited threshold for medical treatments in the United States is $\$ 50,000 /$ QALY [equivalent to approximately $€ 33,885 / \mathrm{QALY}$ (US $\$=€ 0.6777$ )]. It has also been suggested that the implicit threshold for cost effectiveness used by the United Kingdom's National Institute for Clinical Excellence (NICE) is GBP 20,000-30,000/QALY (equivalent to $€ 25,102-37,652 / \mathrm{QALY}$ ). ${ }^{56,57}$ The World Health Organization considers interventions with a cost/QALY of up to three times the country's gross domestic product per capita (approximately $€ 94,000$ for Spain) to be cost effective. ${ }^{58}$ Using these benchmarks, this study demonstrates that 5 years of raltegravir treatment + OBT in treatment-experienced HIVinfected patients in Spain is cost effective (ICER $=€ 31,431$ / QALY). For 3-year and lifetime treatment durations, the ICERs are $€ 22,908$ and $€ 74,468$, respectively.

An assumption of 5 years of raltegravir treatment is relatively conservative; in other economic models for treatmentexperienced patients, durations of treatment with new agents such as enfuvirtide (ENF) and maraviroc were as short as 1-2

\author{
Duration of Tx \\ Time horizon \\ Cost of OBT \\ Discount rate \\ Cost of RAL \\ QoL decrement \\ Ol incidence
}
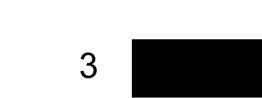
Table 6. Multiway Sensitivity Analysis: Impact on ICER OF VARIATIONS IN CLINICAL AND ECONOMIC INPUTS INTO THE MODEL

\begin{tabular}{llr}
\hline Input & \multicolumn{1}{c}{ Assumption } & $€ / Q A L Y$ \\
\hline Base case & 5 years & 31,431 \\
Source for death rate data & Mocroft et al. ${ }^{4}$ & 33,844 \\
$\begin{array}{l}\text { Source for estimates of } \\
\quad \text { utility weights }\end{array}$ & Stavem et al. ${ }^{18}$ & 28,047 \\
$\begin{array}{l}\text { Source for estimates of } \\
\quad \text { utility weights }\end{array}$ & Schackman et al. ${ }^{47}$ & 42,399 \\
\hline
\end{tabular}

Data reported here are based on an analytic horizon of 50 years.

years. ${ }^{19,59}$ Often the models based treatment duration on "failure" (in other words, patients were taken off a therapy when it failed). This approach was viewed as inappropriate for the raltegravir model, and our results are therefore more conservative than those of other HIV therapies.

When interpreting the benefits attributed to raltegravir, it would be informative to compare them with cost-effectiveness estimates for other recent first-in-class ARTs. Looking specifically at new drugs intended for use in treatment-experienced patients with HIV, ritonavir-boosted tipranavir and ENF are likely comparators. Ritonavir-boosted tipranavir, in the context of a Dutch healthcare system perspective, had an ICER of $€ 42,500 /$ QALY. ${ }^{60}$ ENF represents a new class of drugs, intended as an option for patients suffering treatment exhaustion. Comparison of ENF + OBT vs. OBT alone generated a range of ICERs of $\$ 52,300-168,200 /$ QALY (€35,444-113,989/QALY) where the upper threshold assumed a more intensive treatment. ${ }^{20}$ The ICER for ENF + OBT vs. OBT alone was $\$ 69,500$, assuming patients continued ENF until viral load returned to baseline; for lifetime treatment duration, the ICER was $\$ 158,000$. Another study reported the ICER of ENF as $\$ 24,604 /$ QALY with a treatment duration of 1 year. ${ }^{19}$ The raltegravir ICERs of $€ 31,431 / \mathrm{QALY}$ and $€ 22,908 / \mathrm{QALY}$ for treatment durations of 5 and 3 years compare favorably and are well within the accepted thresholds for health policy makers. Recently, Badia et al. analyzed the cost effectiveness of EN$\mathrm{F}+\mathrm{OBT}$ vs. OBT alone in Spain, ${ }^{61}$ for which they estimated an ICER of $€ 25,082 /$ life-year gained.

The magnitude of the undiscounted survival benefit from OBT (24.7 years) and raltegravir (29.2 years) predicted by the model seems large when compared with those estimated by other studies; for example, Sax et al. reported an undiscounted life expectancy of 5.1 and 6.3 years for patients on OBT and ENF, respectively, ${ }^{19}$ and the respective mean survival estimated by Hornberger et al. is 5.6 and 7.4 years. ${ }^{18}$ Badia et al. predicted a life expectancy of 6.2 years for OBT and 7.8 years for ENF. ${ }^{61}$ It should be noted that there are differences between these models and the raltegravir model. Both Hornberger et al. and Badia et al. estimated life expectancy assuming a time horizon of only 10 years. ${ }^{18,61}$ When we decreased the time horizon in our model from 50 to 10 years, the undiscounted survival benefit from OBT (8.9) and raltegravir ( 9.4 years) predicted by the model fell drastically. The magnitude of increased survival with raltegravir ( 4.5 years) also seems reasonable in light of large reductions in HIV-related mortality as a result of ART. A recent study showed that life expectancy at age 20 years among patients initiating ART increased by more than 13 years (from 36.1 to 49.4 years). ${ }^{62}$
The range of ICER data generated by Sax et al. illustrates another key difficulty in comparing costs between treatments: the clinical and economic assumptions made for each model vary considerably, both within and between models. ${ }^{19} \mathrm{Al}-$ though models cannot take every variable that may influence between-treatment differences for patients into account, sensitivity analyses can be used to gauge likely areas of uncertainty. One variable that had a substantial impact on the raltegravir model was treatment duration. Lower treatment duration of 2-3 years is more typical in cost-effectiveness analyses of antiretrovirals, as current assumptions are that viral resistance limits the duration of efficacy of any treatment. However, with a new class of agent, a longer duration may be sensible, hence our primary focus on a 5-year treatment duration. Evaluation of lifetime treatment duration for raltegravir provided an upper limit for ICER that may be helpful when considering raltegravir in different national and treatment contexts. The raltegravir cost-effectiveness estimates are conservative, as the drug was tested as an add-on to all other HIV therapies, including the highest-priced drugs (such as darunavir, tipranavir, and ENF).

The cost, health, and QoL benefits of raltegravir are based on the premise that raltegravir reduces HIV viral load, leading to higher CD4 cell counts. Patients with higher CD4 cell counts incur lower treatment costs, are less likely to experience OIs, are less likely to die, and have better QoL than patients with lower CD4 cell counts. Patients in the placebo arm of the model also received OBT, and therefore also received some of these benefits. As a result, the incremental benefits of raltegravir over placebo decline as OBT becomes more effective. In addition, because the incidence of OIs and the mortality rates among patients on therapy were also assumed to be low as shown in recent studies, the additional benefits of raltegravir vs. placebo appear lower than they do in cases in which HIV morbidity and mortality were high. Patients on raltegravir also live longer, and can therefore incur more ART costs than those on OBT alone.

This study evaluated the cost effectiveness of raltegravir based on the BENCHMRK trial design (as an add-on to OBT); however, in real world clinical practice raltegravir may replace certain inactive, less tolerable, and/or more expensive agents from OBT, resulting in an even greater cost effectiveness. Due to the trial design, potential benefits generated by the superior safety and tolerability of raltegravir vs. other antiretrovirals could not be incorporated into the model.

\section{Limitations}

This study has several limitations. First, the model is an abstraction of a complex reality involving the uncertain natural history of disease and complicated treatment modalities. In the interest of simplification, we did not explicitly model changes in regimens over time or patient compliance to ART; instead, we assumed that patients will continue on ART for the rest of their lives. This is an important assumption given the sensitivity of the results to assumptions regarding cost of therapy: if expensive, ineffective drugs are excluded, the cost of therapy will be lower, and the cost-effectiveness ratio will improve The potential for future drug availability, the impact of generic drugs (leading to lower ART costs), and the possibility for decreased adherence in real-world situations (also leading to lower yearly ART costs) were not modeled. 
HIV-related indirect medical costs were not available for Spain so the model is based only on direct medical costs.

Second, because the long-term (beyond 48-week) efficacy of raltegravir was not known, virologic failure data for other drugs were used that may not approximate that of raltegravir; this assumption was tested in the sensitivity analysis, however.

Third, multiple sources of data of varying quality were used to estimate the model inputs, which could increase the level of uncertainty in the results. Several sensitivity analyses were conducted in order to evaluate this uncertainty.

Fourth, length of treatment with raltegravir is not known. The base case assumed average treatment duration of 5 years, with viral load transitional probabilities set equally between the placebo and raltegravir groups after that time. Our analysis suggests that the results are sensitive to this assumption. However, other studies have assumed a substantially shorter duration; for example, economic models for maraviroc and ENF as new therapies have had treatment durations as short as 1-2 years, with the duration based on "therapy failure." Such an approach was regarded as inappropriate for the raltegravir model for a number of reasons. First, with highly treatment experienced patients, there may be limited options when a patient fails therapy, with some treatment guidelines such as the British HIV Association (BHIVA) stating that in some cases "when there are a few drug options, it may be better to maintain a therapy on a failing regimen which maximises the fitness effect and has minimal side effects." 63 Second, patients often switch therapies due to intolerance rather than treatment failure. Third, basing treatment duration on virologic failure, arguably, is not as transparent as a simple time cut-off. The latter approach can permit a varying duration of treatment in the sensitivity analysis. ${ }^{64-71}$

Fourth, utility values were conservative, with minimal difference between the most severe and least severe health states. These values were chosen because of the larger sample size of the study, but the lowest utility value was only 0.781 .

Fifth, because of the small size of the different subgroups based on genotypic and phenotypic sensitivity scores and the large number of health states within our model, we could not accurately conduct a robust subgroup comparison within our model.

Finally, despite the significantly higher virologic failure rate in the placebo arm, patients received no further ART costs at failure, because no subsequent lines of ART were incorporated into the model. Higher OBT costs could arguably be added to the placebo arm at failure, since new therapies may be added onto ART regimens, or the regimens themselves may be replaced. Because BENCHMRK subjects were already receiving most of the available therapies for treatmentexperienced patients currently marketed in Spain, it was less confusing to assume that patients in both groups would receive the BENCHMRK trial OBT for the duration of the model. This also meant that it was not necessary to make complex assumptions about the efficacy benefit of adding a new therapy to OBT or about the cost of any new therapies.

\section{Conclusions}

Long-term treatment outcomes with raltegravir, the first drug of a new class of therapy available to treatmentexperienced HIV-infected patients, were projected using a cohort state-transition model. The results suggest that raltegravir can provide substantial survival benefits.

At its current (2008) suggested price of $€ 27.00 /$ day, adding raltegravir to OBT has a favorable ICER vs. placebo when compared with other commonly accepted medical interventions. This conclusion is robust to many changes in the model inputs.

\section{Acknowledgements}

This study was funded by Merck \& Co., Inc. We would like to thank Charlotte Mulcare, Grace Townshend, and Lisa Sullivan, of Watermeadow Medical UK and USA for medical writing and editorial assistance.

\section{Disclosure Statement}

M.A. Chaudhary, R.N. Kumar, G. Nocea, and E. Elbasha are employed by Merck \& Co., which manufactures raltegravir.

\section{References}

1. Centers for Disease Control and Prevention: HIV/AIDS Surveillance Report 2003;15:40. Available at http://www.cdc. gov/hiv/topics/surveillance/resources/reports/2003report/ pdf/2003SurveillanceReport.pdf. Accessed October 6, 2008.

2. Murphy E, Collier A, Kalish L, et al.: Highly active antiretroviral therapy decreases mortality and morbidity in patients with advanced HIV disease. Ann Intern Med 2001;135: 7-26.

3. Little $S$, Holte $S$, Routy $P$, et al.: Antiretroviral-drug resistance among patients recently infected with HIV. N Engl J Med 2002;347:385-394.

4. Mocroft A, Ledergerber B, Viard JP, et al.: Time to virological failure of 3 classes of antiretrovirals after initiation of highly active antiretroviral therapy: Results from the EuroSIDA study group. J Infect Dis 2004;190:1947-1956.

5. Grinsztejn B, Nguyen BY, Katlama C, et al.: Safety and efficacy of the HIV-1 integrase inhibitor raltegravir (MK-0518) in treatment experienced patients with multidrug-resistant virus: A phase II randomized controlled trial. Lancet 2007; 369:1261-1269.

6. Steigbigel RT, Cooper DA, Kumar PN, et al.: Raltegravir with optimized background therapy for resistant HIV-1 infection, N Engl J Med 2008;359:339-354.

7. Cooper DA, Steigbigel RT, Gatell JM, et al.: Subgroup and resistance analyses of raltegravir for resistant HIV-1 infection, N Engl J Med 2008;359:355-365.

8. European Medicines Evaluation Agency, Committee for Medicinal Products for Human Use Summary of Positive Opinion for Isentress: EMEA/CHMP/421343/ 2007. Available at http://www.emea.europa.eu/pdfs/human/press/ pr/52256607 en.pdf. Accessed September 22, 2008.

9. Oliva J, Roa C, and Juan del Llano, JD: Indirect costs in ambulatory patients with HIV/AIDS in Spain: A pilot study. Pharmacoeconomics 2003;21:1113-1121.

10. Freedberg KA, Losina E, Weinstein MC, et al.: The cost effectiveness of combination antiretroviral therapy for HIV disease. N Engl J Med 2001;344:824-831.

11. Weinstein MC, Goldie SJ, Losina E, et al.: Use of genotypic resistance testing to guide HIV therapy: Clinical impact and cost-effectiveness. Ann Intern Med 2001;134:440-450.

12. Weinstein MC, Siegel JE, Gold MR, Kamlet MS, and Russell LB: Recommendations of the Panel on Cost-Effectiveness in Health and Medicine. JAMA 1996;276:1253-1258. 
13. ISPOR 2006 Guidelines on Health Economic Evaluations: Available at http://www.ispor.org/PEguidelines/countrydet. asp? $\mathrm{c}=29 \& \mathrm{t}=1$. Accessed October 8, 2008.

14. Rovira J and Antoñanzas F: Economic analysis of health technologies and programmes. A Spanish proposal for methodological standardisation. Pharmacoeconomics 1995; 8:245-252.

15. Pinto JL and Sánchez Martínez FI: Métodos para la evaluación económica de nuevas prestaciones. Centre De Recerca En Economia I Salut-Cres/ Mimisterio De Sanidad Y Consumo Madrid 2003. Consultado el 03/11/2007 en http://www. sensefums.com/estadEstudios/estadisticas/docs/metodos_ evaluacion.pdf.

16. Cook J, Dasbach E, Coplan P, et al.: Modeling the long-term outcomes and costs of HIV antiretroviral therapy using HIV RNA levels: Application to a clinical trial. AIDS Res Hum Retroviruses 1999;15:499-508.

17. Freedberg KA, Losina E, Weinstein MC, et al.: The costeffectiveness of combination antiretroviral therapy for HIV disease. N Engl J Med 2001;344:824-831.

18. Hornberger J, Green J, Wintfeld N, et al.: Cost-effectiveness of enfuvirtide for treatment-experienced patients with HIV in Italy. HIV Clin Trials 2005;6:92-102.

19. Sax P, Losina E, Weinstein MC, et al.: Cost-effectiveness of enfuvirtide for treatment-experienced patients with advanced HIV disease. J Acquir Immune Defic Syndr 2005; 39:69-77.

20. Kalbfleisch JD and Lawless JF: The analysis of panel data under a Markov assumption. J Am Stat Assoc 1985;80(392): 863-871.

21. Sonnenberg FA and Beck JR: Markov models in medical decision making: A practical guide. Med Decis Making 1993;13:322-331.

22. Grupo de estudio del SIDA (GESIDA), Plan Nacional sobre el SIDA: Prevención de las infecciones oportunistas en pacientes adultos y adolescentes infectados por el VIH. Recomendaciones de GESIDA/Plan Nacional sobre el SIDA. Enferm Infecc Microbiol Clin 2004;22(3):160-176.

23. Instituto Nacional de Estadísitica. Deflactor del IPC: Consultado 18:30 07 Dic 2007 en http://www.ine.es/cgi-bin/ certi? $\mathrm{L}=0 \& \mathrm{PI}=12 \& \mathrm{AI}=2003 \& \mathrm{PF}=02 \& \mathrm{AF}=2007 \& \mathrm{TP}=\mathrm{N}$.

24. Phillips A, Ledergerber B, Horban A, et al.: Rate of viral rebound according to specific drugs in the regimen in 2120 patients with HIV suppression. AIDS 2004;18:1795-1804.

25. Vo TT, Ledergerber B, Keiser O, et al.: Durability and outcome of initial antiretroviral treatments received during 2000-2005 by patients in the Swiss HIV Cohort Study. J Infect Dis 2008;197:1685-1694.

26. Ledergerber B, Lundgren JD, Walker AS, et al.: PLATO Collaboration. Predictors of trend in CD4-positive T-cell count and mortality among HIV-1-infected individuals with virological failure to all three antiretroviral-drug classes. Lancet 2004;364:51-62.

27. EuroSIDA: Tracking HIV/AIDS across Europe 2003. Copenhagen HIV Programme. Hvidovre, Denmark. 2003. Available at http://www.cphiv.dk/Portals /_default/pdf_folder/ EuroSIDA_tracking_2003.pdf. Accessed September 18, 2008.

28. Weinstein MC, Goldie SJ, Losina E, et al.: Use of genotypic resistance testing to guide HIV therapy: Clinical impact and cost-effectiveness. Ann Intern Med 2001;134:440-450.

29. Babiker A, Darbyshire J, Pezzotti P, et al.: CASCADE Collaboration. Changes over calendar time in the risk of specific first AIDS-defining events following HIV seroconversion, adjusting for competing risk. Int J Epidemiol 2002;31:951-958.
30. Hanna D, Gupta L, Jones L, Thompson D, Kellerman S, and Sackoff J: AIDS-defining opportunistic illnesses in the HAART era in New York City. AIDS Care 2007;19:264-272.

31. San-Andrés F-J, Rubio R, Castilla J, et al.: Incidence of acquired immunodeficiency syndrome-associated opportunistic diseases and the effect of treatment on a cohort of 1115 patients infected with human immunodeficiency virus, 1989-1997. Clin Infect Dis 2003;36:1177-1185.

32. Kirk O, Gatell JM, Mocroft A, et al.: Infections with Mycobacterium tuberculosis and Mycobacterium avium among HIV-infected patients after the introduction of highly active antiretroviral therapy. Am J Respir Crit Care Med 2000;162:865-872.

33. Gebo K, Gallant J, Keruly J, and Moore R: Absolute CD4 vs. $\mathrm{Cd} 4$ percentage for predicting the risk of opportunistic illness in HIV infection. J Acquir Immune Defic Syndr 2004;36:1028-1033.

34. Mocroft A, Ledergerber B, Katlama C, et al.: Decline in the AIDS and death rates in the EuroSIDA study: An observational study. Lancet 2003;362:22-29.

35. Olsen $\mathrm{CH}$, Gatell J, Ledergerber B, et al.: Risk of AIDS and death at given HIV-RNA and CD4 cell counts, in relation to specific antiretroviral drugs in the regimen. AIDS 2005;19: 319-330.

36. Moore R and Chaisson R: Natural history of opportunistic disease in an HIV-infected urban clinical cohort. Ann Intern Med 1996;124:633-642.

37. Chaisson R, Gallant J, Keruly J, and Moore R: Impact of opportunistic disease on survival in patients with HIV infection. AIDS 1998;12:29-33.

38. Wohl AR, Lu S, Rollins JN, Simon PA, Grosser S, and Kerndt PR: Comparison of AIDS progression and survival in persons with pulmonary versus extrapulmonary tuberculosis in Los Angeles. AIDS Patient Care STDS 2001;15:463-471.

39. Palella FJ Jr, Baker RK, Moorman AC, et al.: Mortality in the highly active antiretroviral therapy era: Changing causes of death and disease in the HIV outpatient study. J Acquir Immune Defic Syndr 2006;43:27-34.

40. Bonnet F, Lewden C, May T, et al.: Opportunistic infections as causes of death in HIV-infected patients in the HAART era in France. Scan J Infect Dis 2005;37:482-487.

41. Instituto Nacional de Estadística: Tablas de mortalidad de la población de España 1992-2005. Available at http://www. lifetable.de/cgi-bin/Country.plx?Country = Spain. Accessed December 10, 2007.

42. Yip B, Lima VD, Burke TA, Druyts EF, and Hogg RS: Nondrug healthcare resource utilization and costs of managing HIV/AIDS patients prescribed antiretroviral therapy: Evidence from a contemporary HIV practice in Canada. 11th Annual European AIDS Conference (EACS), Madrid, Spain, 24-27 October 2007.

43. Simpson KN, Luo MP, Chumney E, Sun E, Brun S, and Ashraf T: Cost-effectiveness of lopinavir/ritonavir versus nelfinavir as the first-line highly active antiretroviral therapy regimen for HIV infection. HIV Clin Trials 2004;5:294304.

44. Dolan P: Modeling valuations for EuroQoL health states. Med Care 1998;35:1095-1108.

45. Mrus JM, Schackman BR, Wu AW, et al:: Variations in selfrated health among patients with HIV infection. Qual Life Res 2006;15:503-514.

46. Freedberg KA, Scharfstein JA, Seage GR 3rd, et al.: The costeffectiveness of preventing AIDS-related opportunistic infections. JAMA 1998;279:130-136. 
47. Schackman BR, Goldie SJ, Freedberg KA, Losina E, Brazier J, and Weinstein MC: Comparison of health state utilities using community and patient preference weights derived from a survey of patients with HIV/AIDS. Med Decis Making 2002;22:27-38.

48. Stavem K, Frøland SS, and Hellum KB: Comparison of preference-based utilities of the 15D, EQ-5D and SF-6D in patients with HIV/AIDS. Qual Life Res 2005;14:971-980.

49. Lalezari JP, Henry K, O'Hearn M, et al.: Enfuvirtide, an HIV-1 fusion inhibitor, for drug-resistant HIV infection in North and South America. N Engl J Med 2003;348:2175-2185.

50. Clotet B, Bellos N, Molina JM, et al.: Efficacy and safety of darunavir-ritonavir at week 48 in treatment-experienced patients with HIV-1 infection in POWER 1 and 2: A pooled subgroup analysis of data from two randomised trials. Lancet 2007;369:1169-1178.

51. HIV Epidemiology Program, Los Angeles County Department of Health Services: Adult and Adolescent Spectrum of HIV Disease (ASD). Annual Summary Report. January 2004, $1-13$.

52. Keiser O, Taffé $\mathrm{P}$, Zwahlen $\mathrm{M}$, et al.: All cause mortality in the Swiss HIV cohort study from 1990 to 2001 in comparison with the Swiss population. AIDS 2004;18:1835-1843.

53. Egger M, May M, Chêne G, et al.: Prognosis of HIV-1infected patients starting highly active antiretroviral therapy: A collaborative analysis of prospective studies. Lancet 2002;360:119-129.

54. Porter $\mathrm{K}$, Babiker A, Bhaskaran $\mathrm{K}$, et al.: CASCADE Collaboration. Determinants of survival following HIV-1 seroconversion after the introduction of HAART. Lancet 2003;362:1267-1274.

55. El-Sadr WM, Lundgren JD, Neaton JD, et al.; Strategies for Management of Antiretroviral Therapy (SMART) Study Group: CD4 + count-guided interruption of antiretroviral treatment. N Engl J Med 2006;355:2283-2296.

56. Devlin N and Parkin D: Does NICE have a cost-effectiveness threshold and what other factors influence its decisions? A discrete choice analysis. Available at http://www.city.ac .uk/economics/dps/discussion_papers/0301.pdf. Accessed September 18, 2008.

57. Eichler HG, Kong SX, Gerth WC, et al:: Use of costeffectiveness analysis in health-care resource allocation decision-making: How are cost-effectiveness thresholds expected to emerge? Value Health 2004;7:518-528.

58. World Health Organization: Investing in health for economic development. Report of the Commission on Macroeconomics and Health. Geneva, Switzerland, 2001.

59. Kuehne FC, Chancellor J, Mollon P, and Powderly W: Modelling the cost-effectiveness of maraviroc for antiretroviral treatment-experienced individuals. 11th Annual European AIDS Conference (EACS), Madrid, Spain, 24-27 October 2007.

60. Hubben GA, Bos JM, Veltman-Starkenburg CA, et al.: Costeffectiveness of tipranavir versus comparator protease inhibitor regimens in HIV infected patients previously exposed to antiretroviral therapy in the Netherlands. Cost Eff Resour Alloc 2007;5:15.
61. Badia X, Lizán L, Magaz S, Sanz AC, Green J, and Serrano D: Cost-effectiveness analysis of enfuvirtide (ENF) added to an optimized therapy compared with an optimized therapy in patients with HIV/AIDS. HIV Clin Trials 2007;8:235-245.

62. Hogg R, Lima V, Sterne JAC, et al.: Life expectancy of individuals on combination antiretroviral therapy in highincome countries: A collaborative analysis of 14 cohort studies. Lancet 2008;372:293-299.

63. Gazzard B, Bernard AJ, Boffito M, et al.: British HIV Association (BHIVA) guidelines for the treatment of HIV-infected adults with antiretroviral therapy. HIV Med 2006;7:487503.

64. Dye C, Scheele S, Dolin P, Pathania V, and Raviglione MC: Consensus statement. Global burden of tuberculosis: Estimated incidence, prevalence, and mortality by country. WHO Global Surveillance and Monitoring Project. JAMA 1999;282:677-686.

65. Forrest D, Zala C, Djurdjev O, et al.: Determinants of shortand long-term outcome in patients with respiratory failure caused by AIDS-related Pneumocystis carinii pneumonia. Arch Intern Med 1999;159:741-747.

66. Antinori A, Larussa D, Cingolani A, et al:: Prevalence, associated factors, and prognostic determinants of AIDSrelated toxoplasmic encephalitis in the era of advanced highly active antiretroviral therapy. Clin Infect Dis 2004;39: 1681-1691.

67. Hoffmann C, Ernst M, Meyer P, et al.: Evolving characteristics of toxoplasmosis in patients infected with human immunodeficiency virus-1: Clinical course and Toxoplasma gondii-specific immune responses. Clin Microbiol Infect 2007;13:510-515.

68. Kempen JH, Jabs DA, Wilson LA, Dunn JP, West SK, and Tonascia J: Mortality risk for patients with cytomegalovirus retinitis and acquired immune deficiency syndrome. Clin Infect Dis 2003;37:1365-1373.

69. Horsburgh CR Jr, Gettings J, Alexander LN, and Lennox, JL: Disseminated Mycobacterium avium complex disease among patients infected with human immunodeficiency virus, 1985-2000. Clin Infect Dis 2001;33:1938-1943.

70. Vazquez JA, Skiest DJ, Nieto L, et al.: A multicenter randomized trial evaluating posaconazole versus fluconazole for the treatment of oropharyngeal candidiasis in subjects with HIV/AIDS. Clin Infect Dis 2006;42:1179-1186.

71. Centers for Disease Control and Prevention: Treating opportunistic infections among HIV-infected adults and adolescents: Recommendations from CDC, the National Institutes of Health, and the HIV Medicine Association/ Infectious Diseases Society of America. MMWR Recomm Rep 2004;53:1-112.

Address correspondence to: Ritesh N. Kumar

Associate Director, Outcomes Research, Merck \& Co., Inc. One Merck Drive - WS2E-76 Whitehouse Station, New Jersey 08889 E-mail: ritesh_kumar@merck.com 
